\title{
Foundations of Transmathematics
}

\author{
James A.D.W. Anderson \\ Department of Computer Science \\ The University of Reading \\ England \\ RG6 6AY \\ j.anderson@reading.ac.uk
}

Submitted: 18 October 2018

Revised: 28 June 2019

\begin{abstract}
Transmathematics has the ambition to be a total mathematics. Many areas of the usual mathematics have been totalised in the transmathematics programme but the totalisations have all been carried out with the usual set theory, ZFC, Zermelo-Fraenkel set theory with the Axiom of Choice. This set theory is adequate but it is, itself, partial. Here we introduce a total set theory as a foundation for transmathematics.

Surprisingly we adopt naive set theory. It is usually considered that the Russell Paradox demonstrates that naive set theory is incoherent because an apparently well-specified set, the Russell Set, cannot exist. We dissolve this paradox by showing that the specification of the Russell Set admits many unproblematical sets that do not contain themselves and, furthermore, unequivocally requires that the Russell Set does not contain itself because, were it to do so, that one element of the Russell Set would have contradictory membership. Having resolved the Russell Paradox, we go on to make the case that naive set theory is a paraconsistent logic.

In order to demonstrate the sufficiency of naive set theory, as a basis for transmathematics, we introduce the transordinals. The von Neumann ordinals supply the usual ordinals, the simplest unordered set is identical to transreal nullity, and the Russell Set, excluding nullity, is the greatest ordinal, identical to transreal infinity. The generalisation of the transordinals to the whole of established transmathematics is already known.

As naive set theory contains all other set theories, it provides a backwardly compatible foundation for the whole of mathematics.
\end{abstract}




\section{Introduction}

Transmathematics began with an effort to totalise projective geometry for use in computer vision programs [1]. This lead to the totalisation of real arithmetic in transreal arithmetic [7] [10] and of complex arithmetic in transcomplex arithmetic [11]. The topology of the transreal [13] and transcomplex [9] numbers was developed and lead to transreal analysis [12] - all of which confirmed transreal nullity, $\Phi=0 / 0$, as the unique unordered number and the transreal numbers negative infinity, $-\infty=-1 / 0$, and positive infinity, $\infty=1 / 0$, as, respectively, the least and greatest of the ordered numbers. There was some development of paraconsistent logics [8] [14] and Boolean logic was generalised to a wide class of transBoolean logics [10]. A number of applications of transreal arithmetic were discussed in Computer Science [4] [18] [17] [5] [6] [2] and Mathematical Physics [3]. It is a truism to say that all of these developments took place in the usual mathematics; but it required a certain artifice to model the total, trans-systems in the usual, partial mathematics. Our goal, in the present paper, is to extend the foundations of the usual mathematics so that they support total systems in a direct way.

An important result from transreal topology is that nullity is the unique unordered point and infinity is the unique most extremal point [13] [12] [9]. This means that there is exactly one nullity and exactly one infinity so these two numbers are common to the transcomplex, transreal, transrational, transintegral, transnatural and, for our present purposes, transordinal number systems. This justifies calling them, simply, nullity and infinity.

It is possible to have transurreal numbers and other numbers that contain, in the usual sense, transfinite numbers but transreal infinity remains the greatest of them [10]. For our present purposes, this means that transreal infinity, or simply infinity, is the greatest transordinal.

To date, transmathematics has been developed in ZFC - ZermeloFraenkel set theory with the Axiom of Choice. This set theory is adequate to the task but, for our purposes, it has a number of infelicities. Firstly there is no set large enough to be the cardinality of infinity. Secondly the operations of ZFC are partial, not total; for example the set complement does not always exist because there is no universal set. As transreal arithmetic provides a total system of computation, expressing it in a partial system, such as ZFC, gives rise to some inelegance. These problems have been surmounted but they might be avoided by using a set theory that has a universal set.

The set theory NFU - New Foundations with Urelements - provides a universal set and urelements [16][15]. Its operations are still partial, because they do not all apply to urelements or atoms, but this defect is easily remedied. $\mathrm{NFU}$ is entirely adequate for developing the transreal and, specifically, the transordinal numbers, for example by taking the unordered number, nullity, as the set of all atoms and infinity as the set of all sets that have cardinality the universal set. However NFU employs a stratified comprehension which complicates proofs. Ideally we would like a set theory that is as easy to use as ZFC and at least as expressive as NFU. 
Surprisingly we find that naive set theory provides all that is required. It is usually considered that the Russell Paradox demonstrates that naive set theory is incoherent because an apparently well-specified set, the Russell Set, cannot exist. We dissolve this paradox by showing that the specification of the Russell Set admits many unproblematical sets that do not contain themselves and, furthermore, unequivocally requires that the Russell Set does not contain itself as the Russell Element because, were it to do so, that one element of the Russell Set would have contradictory membership.

We observe that each of the von Neumann ordinals do not contain themselves, so they are all members of the Russell Set. It follows, from von Neumann's definition of ordering, that the Russell Set is greater than any von Neumann ordinal and, as the Russell Set has no successor, it is the greatest ordinal - but it contains one element that bars it from being transordinal infinity.

The whole of transreal arithmetic, including the properties of the transreal number nullity, $\Phi=0 / 0$, have been derived, in ZFC, from pairs $\langle n, d\rangle$ where $n$ is the numerator of a transreal fraction, $n / d$, and $d$ is the non-negative denominator [10]. We note that the pair $\Phi=\langle 0,0\rangle$ can be written as the Kuratowski Pair, $\Phi=\{\{\{\}\}\}$, and that $\Phi=\{\{\{\}\}\}$ is the simplest set that is unordered with respect to the von Neumann ordinals. However, nullity does not contain itself so it is a member of the Russell Set and is, therefore, less than the Russell Set. But transreal nullity is required to be unordered with respect to all other transreal numbers so we take transreal infinity equal to the Russell Set, excluding nullity.

Thus we arrive at the transordinals which are composed of: the von Neumann ordinals; the Russell Set, excluding nullity, as infinity; and the simplest unordered set, $\{\{\{\}\}\}$, as nullity.

As the whole of established transmathematics can be derived using the methods in [10], establishing the set of transordinal numbers, together with their ordering, is a sufficient foundation for transmathematics and, by inclusion, for the usual mathematics.

Naive set theory is more expressive than is popularly imagined. Here we develop the transordinals using only pure sets but it is possible for naive set theory to employ any predicate whatsoever, for example predicates describing atoms and classes. Just as the set of all sets can be properly described in naive set theory so, too, the class of all classes can be described. Better still, with an appropriate choice of predicates, all set theories can be described in naive set theory, hence the foundation we offer in the present paper is backwardly compatible with the development of all established mathematics.

It might be supposed that as naive set theory is based on a classical logic, all of its results will be classical, but this is not so. In any set theory with a universal set, objects with contradictory properties and logical gaps occur. We can keep track of the membership of all objects in the universe, either by using pairs of classical truth values or by using metalogical reasoning, but doing so makes naive set theory a paraconsistent logic. Thus we propose a paraconsistent logic as the foundation for all mathematics. 


\section{Naive Set Theory}

Naive Set Theory adopts set-builder notation where $\{x \mid \phi(x)\}$ is syntactic sugar for $\phi(x)$, where $\phi(x)$ is a formula of first-order predicate logic, which evaluates over arbitrarily many terms, $x_{i}$, to give, in typical use cases, a semantic value True, $T$, or else False, $F$; though the formula may result in a contradiction, $T F$; finally the formula may be undecidable or otherwise have no result so that the result is a logical gap, being neither True nor False. When contradictions or gaps occur, in the usual mathematics, it is customary to say that the contradictory or gap object does not exist, but we do not have that freedom when there is a universal set because everything exists somewhere in the universe.

In the jargon of Mathematics, $\{x \mid \phi(x)\}$ is a set with unlimited comprehension. In the jargon of Computer Science, the set $\{x \mid \phi(x)\}$ is provided dynamically by the lazy evaluation or just in time evaluation of the predicate $\phi(x)$. Unlimited comprehension is very expressive; the Universal Set, for example, is given by $V=\{x \mid T\}$. Here the individual $x_{i}$ need not be pure sets, they can be atoms or classes - indeed they can be any mathematical or physical objects whatsoever. Compare with [15].

As usual, sets, such as the set of the first three ordinal numbers, can be enumerated as $\{0,1,2\}$. This is a shorthand for $\{x \mid x=0 \vee x=$ $1 \vee x=2\}$.

\section{Von Neumann Ordinals}

We accept the usual definition of the von Neumann ordinals, including both finite and, in the usual sense, transfinite ordinals. The first few von Neumann ordinals are:

$$
\begin{aligned}
& 0=\{\} \\
& 1=0 \cup\{0\}=\{0\}=\{\{\}\}, \\
& 2=1 \cup\{1\}=\{0,1\}=\{\{\},\{\{\}\}\} \\
& 3=2 \cup\{2\}=\{0,1,2\}=\{\{\},\{\{\}\},\{\{\},\{\{\}\}\}\}, \\
& n=n-1 \cup\{n-1\}=\{0,1,2, \ldots, n-1\}=\{\{\},\{\{\}\}, \ldots,\{\{\},\{\{\}\}, \ldots\}\} .
\end{aligned}
$$

The von Neumann ordinals are ordered by membership so $x<y$ iff $x \in y$. Notice that no von Neumann ordinal contains itself so no von Neumann ordinal is less than itself. In fact all von Neumann ordinals are equal to themselves.

In particular, note that $N=\{\{\{\}\}\}$ is not a von Neumann ordinal so it is unordered with respect to the von Neumann ordinals. Written with just three pairs of set brackets, it is the simplest set that is unordered with respect to the von Neumann ordinals.

In the next section, we find that $N$ is nullity.

\section{Transreal Nullity}

Transreal arithmetic has been derived, in ZFC, from pairs, $\langle n, d\rangle$, where $n$ is the numerator of a transreal fraction, $n / d$, and $d$ is the non-negative 
denominator [10]. The transreal number nullity is given by $\Phi=0 / 0=$ $\langle 0,0\rangle$. Let us re-write this pair as a set, using the Kuratowski Pair $\langle n, d\rangle=$ $\{\{n\},\{n, d\}\}$. Now $\Phi=\langle 0,0\rangle=\{\{0\},\{0,0\}\}=\{\{0\},\{0\}\}=\{\{0\}\}$. Finally writing zero as the von Neumann ordinal, $0=\{\}$, we obtain $\Phi=$ $\{\{\{\}\}\}$. Hence nullity is the simplest unordered von Neumann ordinal, $N$, given in the section immediately above.

We now adopt a definition of nullity, relative to the von Neumann ordinals. In the Discussion we consider the definition of nullity relative to other models of the ordinals but we prefer the present definition.

Definition 1 (Nullity) When the ordinals are given by the von Neumann ordinals, nullity is the set $\Phi=\{\{\{\}\}\}$.

\section{The Russell Paradox}

The Russell Paradox concerns the construction of a particular object, called the Russell Set. We adopt the usual definition of the Russell Set as given in set-builder notation.

Definition 2 (The Russell Set) The Russell Set, $R$, is given by $R=$ $\{x \mid x \notin x\}$.

In order to facilitate discussion, we add subscripts to the definition: $R_{s}=\left\{x_{1} \mid x_{2} \notin x_{3}\right\}$, where $x_{1}=x_{2}=x_{3}$. We also introduce the term Russell Element, $R_{e}$, to distinguish the non-paradoxical use of the Russell Set, $R_{s}$, as a set, from its paradoxical use as an element, $R_{e}$. That is, the Russell Set and the Russell Element are distinct names for the same mathematical object. These names are sensitive to the context of whether the object is being used as a set or as an element, just as the subscripted $x_{1}, x_{2}, x_{3}$ are sensitive to their role in the definition of the Russell Set. Using context sensitive names greatly clarifies the discussion and is to be recommended in similar circumstances.

Suppose $x_{1}$ is the von Neumann ordinal zero, then $x_{1}=0$ is in $R_{s}$ because $x_{2}=0 \notin x_{3}=0$ and similarly for all von Neumann ordinals. Thus far in its lazy evaluation, $R_{s}$ is a very high cardinality set that contains all finite and, in the usual sense, transfinite von Neumann ordinals.

Now we ask the paradoxical question: is $R_{e}$ in $R_{s}$ ? Suppose $x_{1}=R_{e}$, then we are supposing $R_{e} \in R_{s}$, whence $R_{e} \notin R_{s}$, by $x_{2} \notin x_{3}$, with $x_{2}=$ $R_{e}$ and $x_{3}=R_{s}$. That is: $R_{e} \in R_{s} \Longrightarrow R_{e} \notin R_{s}$. Conversely suppose that $x_{2}=R_{e}$ and $x_{3}=R_{s}$, then we are supposing $R_{e} \notin R_{s}$, whence $R_{e} \in R_{s}$, by $x_{1}=R_{e}$. That is: $R_{e} \notin R_{s} \Longrightarrow R_{e} \in R_{s}$. Combining these two implications we have the usual paradoxical bi-implication: $R_{e} \in$ $R_{s} \Longleftrightarrow R_{e} \notin R_{s}$.

Now we ask, what can satisfy this paradoxical bi-implication? It cannot be $x_{1} \in R_{s}=T$ or $x_{1} \in R_{s}=F$ but suppose $x_{1} \in R_{s}$ is the contradiction $x_{1} \in R_{s}=T F$. Now we can choose $R_{e} \in R_{s}=T \Longrightarrow R_{e} \notin R_{s}=F$ and, conversely, $R_{e} \in R_{s}=F \Longrightarrow R_{e} \notin R_{s}=T$. Thus the paradoxical implication can be satisfied by the contradictory fact that $R_{e}$ is and is not in $R_{s}$. But, by the Law of the Excluded Middle, we are not permitted contradictions in the first-order predicate calculus employed by 
set-builder notation, therefore the Russell Element, $R_{e}$, is outlawed from being a member of the the Russel set, $R_{s}$.

Thus we see that the Russel Set contains many elements, because it contains all of the finite and, in the usual sense, transfinite von Neumann ordinals. In addition, the Russell Set contains many sets that are not von Neumann ordinals, for example it contains the set $\Phi=\{\{\{\}\}\}$. But the Russell Set unequivocally does not contain the Russell Element because, were it to do so, that would introduce an illegal contradiction. Put another way, if the Russell Set had an element that was the Russell Element then the Russell Set would not be a set, it would be a contradictory class. Critically, by insisting that the Russell Set is a set, we unequivocally require that the Russell Element is not an element of the Russell Set. Put yet another way, the Russell Element is not in the Russell Set. This justifies us in distinguishing the Russel Set, which is a set excluding the Russell Element, from the Russell Element itself, which is a contradictory class. Both the Russell Element and the Russell Set are in the Universal Set, $V$.

\section{Transreal Infinity}

Transreal infinity must satisfy two requirements. Firstly it must be the greatest of the ordered numbers. Secondly it must be unordered with respect to nullity. Furthermore nullity must be unordered with respect to all other transreal numbers. This last requirement is already satisfied for the ordinal numbers: by construction, nullity is unordered with respect to the von Neumann ordinals.

We now define transreal infinity so that nullity is unordered with respect to it.

Definition 3 (Infinity) When the ordinals are given by the von Neumann ordinals, infinity is given by $\infty=\{x \mid x \notin x\} \backslash\{\Phi\}$.

No ordinal is less than itself so every ordinal is a member of infinity. Hence transordinal infinity is greater than every ordinal, making it the greatest transordinal, ignoring nullity which is unordered.

The fact that infinity is the only transordinal greater than the ordinals means that it is the least such number and is, therefore, the ordinal type of the ordinals. As transordinal infinity has no successor, it seems reasonable to define that it is its own ordinal type.

\section{Transordinals}

We are now in a position to define the transordinals.

Definition 4 (Transordinals) Let $\mathcal{O}$ be the set of ordinals then the set of transordinals is given by $\mathcal{O}^{T}=\mathcal{O} \cup\{\infty\} \cup\{\Phi\}$. 


\section{Discussion}

We have described infinity and nullity as pure sets but we might have to make different choices for these numbers if we choose a different model of the ordinals. For example, if we choose the Zermelo ordinals, $0=\{\}$, $1=\{\{\}\}, 2=\{\{\{\}\}\}, \ldots$ then we could not use $\{\{\{\}\}\}$ as nullity because this is the Zermelo ordinal 2. In this case we could use $\{\{\},\{\{\}\}\}$ as nullity, which is the von Neumann ordinal 2 . We could choose any of very many models of the ordinals, infinity and nullity but we prefer the model presented here, partly because of the convenience of using von Neumann ordinals, partly because of the happy coincidence that $\Phi=0 / 0=\langle 0,0\rangle=$ $\{\{\{\}\}\}$ and because $\{\{\{\}\}\}$ is the simplest unordered set. This is a great deal of mathematical structure that might support future developments.

It is surprising that we are able to dissolve the Russell Paradox. We are prompted to ask why this is the case? Is there some irreparable flaw in our argument so that the dissolution has not actually occurred? If this is the case, the sooner we become aware of such an error, the better! Are there genuinely two choices of how to resolve the paradox - either by disallowing the Russell Set or by disallowing the Russell Element? If this is the case, we would like to identify an axiomatic basis for each choice. Is the usual interpretation of the Russell Set mistaken? If this is the case, we would like to know how the mistake occurred and what it is about the present paper that exposes it? Is it our use of context sensitive names or of lazy evaluation? Is it our familiarity with paraconsistent logics or Universal Sets? There are many aspects of our handling of the Russell Paradox that might bear mathematical, historical and philosophical analysis.

Let us expand on the importance of universal sets. A universal set is a set of everything. Depending on an author's metaphysical inclinations it may contain all sets, all atoms, all physcial objects, anything whatsoever. This has an important consequence. In the usual mathematics, contradictory objects, such as the set that is not an element of the Universal Set, are said not to exist. Consequently the usual mathematics is partial, it allows that certain objects do not exist and that certain questions have no answer. By contrast, transmathematics aims to develop total systems - it does not allow the non-existence of anything and insists that every question has an answer. The question is, how can we envisage doing this?

There are two ways. One is for transmathematics to be concerned with formulas stored in sets. These are evaluated, mechanically or by employing mathematical intuition, to see what they describe: they may describe sets, atoms, contradictory classes or anything at all. The second way is to keep track of the regions in the universe where objects lie.

Consider a usual set. Let us say that all of its elements occur in the interior of the set and all of the elements in its complement occur in the exterior of the set. If we enforce the Law of the Excluded Middle then we need one bit, that is one classical truth value, True or else False, to keep track of where an object lies - either it is in the interior or else the exterior of the set. But if we do not enforce the Law of the Excluded Middle then we need two bits. Let us arrange these two bits as a pair, $\langle I, E\rangle$, where $I$ encodes membership of the interior of a set and $E$ encodes membership of the exterior of a set. Now $\mathcal{T}=\langle T, F\rangle$ encodes that it is True that the 
object lies only in the interior of the set. The symbol $\mathcal{T}$ indicates that it is classically True that the object is in the set. Conversely, $\mathcal{F}=\langle F, T\rangle$ encodes that it is True that the object lies only in the exterior of the set. The symbol $\mathcal{F}$ indicates that it is classically False that the object is in the set. There are two more cases to consider. The contradictory case, $\mathcal{C}=\langle T, T\rangle$, indicates that it is a classical contradiction, both True and False, that the object lies in the set. We shall say that all contradictory elements lie in the border of a set. Thus the Russell Element lies in the border of the Russell Set, similarly the set that is not an element of the Universal Set lies in the border of the Universal Set. Finally the case of a logical gap, $\mathcal{G}=\langle F, F\rangle$ indicates that it is neither True nor False that the object lies in the set. Where then, does it lie? We shall say that it lies in the background of the set. Thus we equip every set with four distinct regions: an interior, exterior, border, and background. Now a usual or classical set has an empty border; an antinomy has a non-empty border; and the Universal Set has both an empty exterior and an empty background, it partitions all elements into its interior and border. If we try to insist that a set lies in the exterior or background of the Universal Set, we find that it actually lies in the Universal Set's border, making the Universal Set an antinomy, not a set. We might like to call the Universal Set the Universal Antinomy or the Universal Class, but our four valued logic, corresponding to four regions, is a very long way from the usual notion of a class. We prefer to say that we are dealing with a transclass. Then $V$ is the Universal Transclass, the object given in Definition 2 is the Russell Transclass that turns out to be the antinomy, we may call it the Russell Antinomy, that we have been calling the Russell Element, and the interior of this transclass is the set we have been calling the Russell Set.

Mathematicians might like to adopt the Axiom of Logical Omniscience so that the putative transclass theory is monotonic. Practical scientists, however, are likely to prefer that it is non-monotonic. They are likely to allow, for example, that they might discover that a proposition is True and, later, that it is False: at which time they will conclude that it is a Contradiction. Having discovered this fact, they might go on to discover that the proposition is a Gap. At any point they might adjust their axioms so that the proposition is classically True or else False.

We envisage that the putative transclass theory will provide a foundation for transmathematics that is, itself, founded upon naive set theory which, in turn, is founded on first-order predicate logic. Until such time as we develop a suitable transclass theory, we will use naive set theory as a foundation for transmathematics.

Now let us say something about paraconsistency. In classical logic, all theorems follow from a contradiction. The system is said to blow up - it admits everything. This is not useful so classical systems aim to retain consistency. By contrast, paraconsistent logics do not blow up: only some, not all, theorems follow from a contradiction. This raises the question, is the usual mathematics consistent or paraconsistent? Certainly the usual mathematics aims to be consistent. It adopts the Axiom of the Excluded Middle, which outlaws contradictions, and it adopts logical type systems that prevent inconsistent deductions. But very large parts of the usual mathematics also uses Proof by Contradiction. This proof 
method explicitly constructs a contradiction and then deduces that some contradictory object does not exist. By saying that the contradictory object does not exist, the usual mathematics removes that object from the domain of discourse, we would say, places it in the background, and this prevents blowing up. Proofs by contradiction are used, very widely, in the usual mathematics, including set theories, so the usual mathematics and set theories are, in this way, paraconsistent. In particular, naive set theory is paraconsistent.

As has been said, above, naive set theory and any set theory with a universal set or class is paraconsistent by virtue of admitting contradictory objects into the universe. In the present paper, we have shown that naive set theory can admit the Russell Antinomy, as a contradictory object, and yet we can reason about its elements and use the interior of the Russell Antinomy to construct useful objects - the Russell Set and transreal infinity - all without blowing up. This is yet more evidence that naive set theory is paraconsistent, despite historical attempts to disallow inconsistent objects.

It may be of philosophical interest that naive set theory is paraconsistent, as is a very large part of the usual mathematics.

Our interest in total systems gives us a very wide remit. We are not only concerned with human reasoning but also with computer reasoning. In computational systems, totallity is hugely beneficial. For example, it allows a computer to try things at random, without the risk of crashing. In our view, naive set theory and the putative transclass theory provide a total and very expressive system that is entirely satisfactory for our foreseeable needs.

We leave the last word to Alan Turing [19]:

The processes of inference used by the machine need not be such as would satisfy the most exacting logicians. There might for instance be no hierarchy of types. But this need not mean that type fallacies will occur, any more than we are bound to fall over unfenced cliffs. Suitable imperatives (expressed within the systems, not forming part of the rules of the system) such as "Do not use a class unless it is a subclass of one which has been mentioned by teacher" can have a similar effect to "Do not go too near the edge."

\section{Conclusion}

We cover a lot of ground in this short paper. We dissolve the Russell Paradox, thereby restoring naive set theory to its role as a coherent and total set theory that is a sufficient foundation for all mathematics, including transmathematics. We use this set theory to construct the transordinal numbers, including infinity and nullity, as pure sets. We note that naive set theory is a paraconsistent logic and we propose developing a transclass theory on it that operates on pairs of classical truth values to describe the interior, exterior, border and background of a transclass. 


\section{Acknowledgements}

Many years ago, Walter Gomide asked the seminal question, which set is nullity? This question finally has an answer: when the ordinals are given by the von Neumann ordinals, nullity is the set $\Phi=\{\{\{\}\}\}$ and infinity is the Russell Set excluding nullity.

Thanks are due both to Walter Gomide and to Tiago dos Reis for many helpful discussions on paraconsistent logics and set theories.

\section{References}

[1] J. A. D. W. Anderson. Representing geometrical knowledge. Phil. Trans. Roy. Soc. Lond. Series B., 352(1358):1129-1139, 1997.

[2] J. A. D. W. Anderson. Transmathematical basis of infinitely scalable pipeline machines. International Conference On Computational Science, pages 1828-1837, 2015.

[3] J. A. D. W. Anderson and T. S. dos Reis. Transreal newtonian physics operates at singularities. Synesis, 7(2):57-81, 2015.

[4] James A. D. W. Anderson. Perspex machine vii: The universal perspex machine. In Longin Jan Lateki, David M. Mount, and Angela Y. $\mathrm{Wu}$, editors, Vision Geometry XIV, volume 6066 of Proceedings of SPIE, pages T1-T17, 2006.

[5] James A. D. W. Anderson. Trans-floating-point arithmetic removes nine quadrillion redundancies from 64-bit ieee 754 floating-point arithmetic. In Lecture Notes in Engineering and Computer Science: Proceedings of The World Congress on Engineering and Computer Science 2014, WCECS 2014, 22-24 October, 2014, San Francisco, USA., volume 1, pages 80-85, 2014.

[6] James A. D. W. Anderson and Tiago S. dos Reis. Transreal limits expose category errors in ieee 754 floating-point arithmetic and in mathematics. In Lecture Notes in Engineering and Computer Science: Proceedings of The World Congress on Engineering and Computer Science 2014, WCECS 2014, 22-24 October, 2014, San Francisco, USA., volume 1, pages 86-91, 2014.

[7] James A. D. W. Anderson, Norbert Völker, and Andrew A. Adams. Perspex machine viii: Axioms of transreal arithmetic. In Longin Jan Lateki, David M. Mount, and Angela Y. Wu, editors, Vision Geometry XV, volume 6499 of Proceedings of SPIE, pages 2.1-2.12, 2007.

[8] James A.D.W. Anderson and Walter Gomide. Transreal arithmetic as a consistent basis for paraconsistent logics. In Lecture Notes in Engineering and Computer Science: Proceedings of The World Congress on Engineering and Computer Science 2014, WCECS 2014, 22-24 October, 2014, San Francisco, USA., volume 1, pages 103-108, 2014.

[9] T. S. dos Reis and J. A. D. W. Anderson. Transcomplex topology and elementary functions. In S. I. Ao, Len Gelman, David W. L. Hukins, Andrew Hunter, and A. M. Korsunsky, editors, World Congress on Engineering, volume 1, pages 164-169, 2016. 
[10] T. S. dos Reis, W. Gomide, and J. A. D. W. Anderson. Construction of the transreal numbers and algebraic transfields. IAENG International Journal of Applied Mathematics, 46(1):11-23, 2016.

[11] Tiago S. dos Reis and James A. D. W. Anderson. Construction of the transcomplex numbers from the complex numbers. In Lecture Notes in Engineering and Computer Science: Proceedings of The World Congress on Engineering and Computer Science 2014, WCECS 2014, 22-24 October, 2014, San Francisco, USA., volume 1, pages 97-102, 2014.

[12] Tiago S. dos Reis and James A. D. W. Anderson. Transreal calculus. IAENG International Journal of Applied Mathematics, 45(1):51-63, 2015.

[13] Tiago S. dos Reis and James A. D. W. Anderson. Transreal limits and elementary functions. In Haeng Kon Kim; Mahyar A. Amouzegar; Sio long Ao, editor, Transactions on Engineering Technologies - World Congress on Engineering and Computer Science 2014, pages 209-225. Springer-Verlag, 2015.

[14] Walter Gomide, Tiago S. dos Reis, and James A. D. W. Anderson. Transreal logical space of all propositions. In Transactions on Engineering Technologies - World Congress on Engineering and Computer Science 2014, number To appear. Springer-Verlag, 2015.

[15] Randall Holmes. Elementary Set Theory with a Universal Set, volume 10 of Cahiers du Centre de Logique. Academia, Louvain-laNeuve (Belgium), 1998.

[16] Ronald Björn Jensen. On the consistency of a slight (?) modification of quine's "new foundations". Synthese, 19(1/2):250-264, December 1968.

[17] C. J. A. Kershaw and James A. D. W. Anderson. Perspex machine vi: A graphical user interface to the perspex machine. In Longin Jan Lateki, David M. Mount, and Angela Y. Wu, editors, Vision Geometry XIV, volume 6066 of Proceedings of SPIE, pages S1-S10, 2006.

[18] M. P. Spanner and James A. D. W. Anderson. Perspex machine v: Compilation of c programs. In Longin Jan Lateki, David M. Mount, and Angela Y. Wu, editors, Vision Geometry XIV, volume 6066 of Proceedings of SPIE, pages A1-A12, 2006.

[19] A. M. Turing. Computing machinery and intelligence. Mind, 49:433460, 1950. 